\title{
Detecção de oocistos de Cryptosporidium spp. e cistos de Giardia spp. em amostras de esgoto bruto ou tratado: avaliação crítica dos métodos
}

\section{Detection of Cryptosporidium spp. 0ocysts and Giardia spp. cysts in raw and effluent wastewater: critical evaluation of methods}

\author{
Luciana Urbano dos Santos \\ Bióloga. Pesquisadora do Departamento de Saneamento e Ambiente da Faculdade de Engenharia Civil, Arquitetura e Urbanismo da \\ Universidade Estadual de Campinas (Unicamp)
}

Romeu Cantusio Neto

Biólogo. Laboratório de Microbiologia da Sociedade de Abastecimento de Água e Saneamento de Campinas (Sanasa)

Regina Maura Bueno Franco

Bióloga. Professora do Departamento de Biologia Animal do Instituto de Biologia da Unicamp

José Roberto Guimarães

Químico. Professor do Departamento de Saneamento e Ambiente da Faculdade de Engenharia Civil, Arquitetura e Urbanismo da Unicamp

\section{Resumo}

Neste trabalho, avaliou-se a eficiência dos métodos centrífugo-concentração e filtração em membrana, na detecção de oocistos de Cryptosporidium spp. e cistos de Giardia spp. em amostras de esgoto bruto e tratado, provenientes de um sistema de lodos ativados (estação de tratamento de esgoto, Samambaia, Campinas, em São Paulo). As amostras foram coletadas quinzenalmente por dois anos: 53 amostras de esgoto bruto (AFL), 53 de efluente tratado sem desinfecção por luz ultravioleta (EFL) e 38 de efluente tratado e desinfetado por luz ultravioleta (EFL+UV). Cistos de Giardia spp. foram encontrados em $90,5 \%$ das amostras AFL; em 96,2\%, de EFL; e em 94,7\%, de EFL+UV. Oocistos de Cryptosporidium spp. foram detectados em 6,4\% das amostras AFL e em 2,6 \% de EFL+UV. Ambos os métodos mostraram-se eficientes na detecção destes protozoários em todos os tipos de amostras, além de apresentarem baixo custo por análise.

Palavras-chave: doenças de veiculação hídrica; parasitoses; protozoários.

\section{Abstract}

In this study, the efficiency of centrifuge-concentration and membrane filtrated methods was evaluated in the detection of Cryptosporidium spp. oocysts and Giardia spp. cysts in raw or treated wastewater samples, from activated sludge systems (ETE - Samambaia, Campinas, in São Paulo). The samples were collected once a fortnight for two years: 53 samples of influent (AFL), 53 samples of treated effluent without ultraviolet disinfection (EFL), and 38 samples of treated effluent with ultraviolet disinfection (EFL+UV). Giardia spp. cysts were found in 90.5\% of the AFL samples; in 96.2\% of the samples, EFL; and in 94.7\%, EFL+UV. Cryptosporidium spp. oocysts were detected in $6.4 \%$ of AFL samples and $2.6 \%$ of EFL+UV. Both methods showed efficiency when detecting protozoa in all types of samples, besides having low costs by analysis.

Keywords: waterborne diseases; parasitoses; protozoa 


\section{Introdução}

A lista dos agentes patogênicos à saúde humana é vasta, compreendendo vírus, bactérias, fungos, protozoários e helmintos (NITHIUTHAI et al., 2004; OTTOSON et al., 2006; NORDGREN et al., 2009). As formas infectantes destes agentes são liberadas via fezes ou urina dos hospedeiros infectados, fazendo com que o esgoto doméstico seja uma das mais importantes fontes de contaminação ambiental e com grande impacto na saúde pública (MACPHERSON, 2005; MONTEMAYOR et al., 2005).

Dados da Organização Mundial da Saúde (OMS) apontam que $88 \%$ das mortes por diarreia em todo o mundo, são causadas por ingestão de água contaminada ou saneamento inadequado. Das 1,8 milhões de pessoas que vão ao óbito por ano em função desta patogenia, 90\% são crianças com menos de cinco anos de idade (ONU, 2006). Avaliações moleculares das amostras colhidas em surtos de doenças de veiculação hídrica evidenciaram que o esgoto doméstico foi a principal fonte de contaminação dos mananciais (MACPHERSON, 2005; AIYUK et al., 2006; OTTOSON et al., 2006; LI et al., 2009).

Entre os vários grupos de organismos patogênicos que podem causar gastroenterite no homem, estão os protozoários Cryptosporidium spp. e Giardia spp., os quais têm sido frequentemente detectados em amostras ambientais em todo o mundo, incluindo no Brasil (FRANCO et al., 2001; HACHICH et al., 2004, SANTOS et al., 2004; CANTUSIO-NETO et al., 2006; LEAL et al., 2008). Estima-se que $20 \%$ da população mundial esteja parasitada com Giardia spp. (ROCKWELL, 2007). O Cryptosporidium spp. é apontado como um dos principais agentes causadores de diarreia em crianças (ROSE et al., 2002), sendo particularmente preocupante em indivíduos imunocomprometidos, nos quais a taxa de mortalidade muda de 0,001\% para aproximadamente 60,0\% (BOULTER-BITZER et al., 2007).

No Brasil, a ocorrência de oocistos de Cryptosporidium spp. e cistos de Giardia spp. é relatada em amostras de água superficial e de esgoto, bruto e tratado, sendo frequentemente detectados numa ampla faixa de concentração (DIAS JÚNIOR 1999; FARIAS et al., 2002; HACHICH et al., 2004; CANTUSIO NETO et al., 2010). Santos et al. (2004) analisaram dois métodos de concentração para amostras de esgoto em etapas de purificação com éter ou solução saturada de sacarose. Após encontrarem concentrações de oocistos e cistos na ordem de $10^{4}$ e $10^{6}$ formas/L, respectivamente, os autores concluíram que nos métodos em que se realiza menor número de etapas, há melhor recuperação dos protozoários. A técnica de centrifugação, seguida de clarificação com éter, mostrou-se mais eficiente na recuperação de cistos quando comparada com o método de purificação por flutuação em sacarose. Enquanto que, na primeira, são realizadas apenas três lavagens (n = 3), na segunda este número é mais elevado ( $n=5)$, resultando, consequentemente, numa maior possibilidade de perda dos organismos durante o procedimento.

Os principais objetivos do presente trabalho são: apresentar e discutir a aplicabilidade do método de centrífugo-concentração (CC) para amostras de afluente, e filtração em membrana (FM) para amostras de efluente de uma estação de tratamento de esgoto (ETE), na recuperação de oocistos de Cryptosporidium spp. e cistos de Giardia spp.

\section{Material e métodos}

Na ETE Samambaia, localizada em Campinas, na cidade de São Paulo e que está sendo avaliada no presente trabalho, é tratado o esgoto doméstico (vazão $70 \mathrm{~L} /$ segundos) pelo sistema de lodos ativados. Apenas para experimentação, na saída da ETE foi instalado um reator com duas lâmpadas ultravioletas, modelo InLine 250, Sistema de Desinfecção ultravioleta (UV) de Líquidos - Alta Intensidade (Germetec UV \& IR Technology Ltda ${ }^{\circledR}$ ), operando numa vazão de $15 \mathrm{~L} / \mathrm{s}$ e com uma dose de UV entre 25 e $30 \mathrm{mWs} \mathrm{cm}^{-2}$.

Foram analisados três diferentes tipos de amostras colhidas da ETE: i) afluente (esgoto bruto) designado AFL; ii) efluente (esgoto tratado) e não-desinfetado com UV, designado EFL e iii) efluente desinfetado com UV, designado EFL+UV. O tempo de detenção hidráulica (TDH) do esgoto era próximo de 50 horas (desde a entrada do afluente na ETE até o lançamento do efluente no córrego Samambaia); portanto, as coletas do afluente e efluente foram realizadas levando-se em consideração o TDH, com a finalidade de serem obtidas relações mais confiáveis de eficiência. As amostras foram colhidas em frascos plásticos, os quais foram previamente descontaminados e lavados com solução de eluição (Tween $80-0,1 \%$ ), para evitar a adesão de oocistos e cistos na parede do frasco e foram mantidas em $10^{\circ} \mathrm{C}$ antes de serem processadas.

As amostras do afluente foram concentradas e purificadas conforme o protocolo adaptado de Robertson et al. (2000): $5 \mathrm{~mL}$ de afluente previamente filtrados em tela $\left(1 \mathrm{~mm}^{2}\right)$ foram colocados em tubos de centrífuga $(15 \mathrm{~mL})$, e o volume total do tubo foi completado com solução de eluição. Os tubos foram centrifugados a $1.500 \mathrm{x} \mathrm{g}$ (15 minutos), o sobrenadante descartado e o sedimento final foi transferido para um tubo de microcentrifugação. O efluente desinfetado ou não com UV (mínimo de $1.200 \mathrm{~mL}$ ) foi avaliado de acordo com o protocolo de Franco et al. (2001): as amostras foram filtradas em membranas de ésteres de celulose (Millipore ${ }^{\circledR}$ ), com $47 \mathrm{~mm}$ de diâmetro e porosidade nominal de 3 $\mu \mathrm{m}$. Este procedimento foi feito com um sistema de filtração com bomba à vácuo e fluxo de $4 \mathrm{~L} /$ minutos em porta filtro da marca Gelman ${ }^{\circledR}$. Após a transferência das membranas para placas plásticas estéreis, foi feita a etapa de recuperação dos cistos e oocistos 
por extração mecânica e lavagens da superfície da membrana por 20 minutos, alternadamente, com solução de eluição. O líquido resultante foi concentrado por centrifugação $(1.050 \mathrm{x} g$ por dez minutos), o sobrenadante aspirado e o sedimento resultante foi novamente lavado e centrifugado. Uma alíquota de $5 \mu \mathrm{L}$ do sedimento final foi analisada por reação de imunofluorescência direta (RID) e confirmada pelo corante vital DAPI.

Foram realizados experimentos controles negativo e positivo. Utilizando-se o teste $t$ de Student, foi verificado se havia uma diferença significativa entre as concentrações de oocistos e cistos encontradas entre os três diferentes tipos de amostras analisadas.

\section{Resultados e discussão}

Nos experimentos controles negativo realizados, nenhum oocisto ou cisto foi detectado, indicando que não houve contaminação durante todo o processo de filtração e eluição das membranas ou confecção das lâminas. É importante que estas avaliações sejam realizadas entre os processamentos das amostras ou mesmo intercaladas com os experimentos controle-positivo, pois caso haja falha, essa será facilmente detectada evitando que resultados falso-positivos sejam obtidos.

Em relação aos experimentos controles-positivo, utilizando-se a técnica de FM, foram obtidas taxas de recuperação de 26,6\% para oocistos e 58,8\% para cistos. Quando a metodologia de CC foi empregada, foram observados valores mais elevados: 65,8\% para oocistos de Cryptosporidium spp. e 124,0\% para cistos de Giardia spp. Níveis de recuperação superiores a $100 \%$ podem ocorrer e isto está relacionado à padronização do inóculo utilizado na avaliação. A enumeração das formas previamente à diluição e inoculação não são passos muito simples, pois aspectos biológicos como idade e origem dos organismos, procedimentos prévios de limpeza, o meio e o tempo de armazenamento da suspensão podem influenciar na agregação das formas e, consequentemente, nos valores de recuperação (BUKHARI et al., 1998; LINDQUIST et al., 1999).

A realização de experimentos controles-positivos é imprescindivel para atestar a sensibilidade do método escolhido. Previamente à sua condução, deve-se investigar se a amostra está naturalmente contaminada, ou não, com oocistos ou cistos. Para tanto, existem kits disponíveis comercialmente, sendo que os protozoários que compõem o inóculo são marcados com sulforodamina, um corante fluorogênico que, quando excitado por uma radiação de comprimento de onda específico do microscópio de epifluorescência, permite que os organismos sejam visualizados em vermelho, diferente do Fluorescein isothiocyanate (FITC) da RID, no qual os organismos são visualizados na coloração verdemaçã brilhante. Isto possibilita ao microscopista a diferenciação entre organismos naturalmente presentes na amostra (verde-maçã brilhante) daqueles inoculados artificialmente (vermelho). Desta forma, o controle-positivo pode ser realizado junto com o monitoramento da amostra, em um único processamento, porém, o custo do kit é alto. No presente estudo, optou-se por avaliar uma alíquota da amostra bruta e subtrair os organismos encontrados da contagem realizada para a taxa de recuperação.

Durante os dois anos de avaliação, oocistos de Cryptosporidium spp. foram detectados em: i) 6,4\% das amostras de AFL com uma concentração média de $6,00 \times 10^{4}$ oocistos/L e ii) uma amostra de EFL+UV (2,6\% das amostras) com concentração de $1,60 \times 10^{2}$ oocistos/L. Entretanto, cistos de Giardia spp. foram frequentemente detectados nos três tipos de amostras em concentrações superiores às encontradas para oocistos (Tabela 1).

Uma diferença significativa $(\mathrm{p}=0,01)$ foi obtida entre as médias do número de cistos de Giardia spp. observado nas amostras de AFL e de EFL e EFL+UV. Entretanto, não houve diferença estatística entre as médias do número de cistos $(\mathrm{p}=0,01)$ para as amostras do EFL e EFL+UV. Este resultado indica a reprodutibilidade

Tabela 1 - Número médio de cistos de Giardia spp. por litro durante os quatro semestres de avaliação em amostras da ETE - Samambaia

\begin{tabular}{|c|c|c|c|c|}
\hline Amostras & $2^{\circ}$ semestre de 2003 & $1^{\circ}$ semestre de 2004 & $2^{\circ}$ semestre de 2004 & $1^{\circ}$ semestre de 2005 \\
\hline Afluente (AFL) & $\begin{array}{c}1,20 \times 10^{5} \\
\pm 9,14 \times 10^{4}\end{array}$ & $\begin{array}{c}1,06 \times 10^{5} \\
\pm 6,45 \times 10^{4}\end{array}$ & $\begin{array}{c}1,34 \times 10^{5} \\
\pm 1,01 \times 10^{5}\end{array}$ & $\begin{array}{c}7,38 \times 10^{4} \\
\pm 8,13 \times 10^{4}\end{array}$ \\
\hline Efluente (EFL) & $\begin{array}{c}1,73 \times 10^{3} \\
\pm 1,09 \times 10^{3}\end{array}$ & $\begin{array}{c}1,24 \times 10^{3} \\
\pm 1,71 \times 10^{3}\end{array}$ & $\begin{array}{c}9,13 \times 10^{2} \\
\pm 4,33 \times 10^{2}\end{array}$ & $\begin{array}{c}6,78 \times 10^{2} \\
\pm 5,37 \times 10^{2}\end{array}$ \\
\hline Efluente (EFL+UV) & $\begin{array}{c}3,12 \times 10^{3} \\
\pm 1,12 \times 10^{3}\end{array}$ & $\begin{array}{c}6,41 \times 10^{2} \\
\pm 5,89 \times 10^{2}\end{array}$ & $\begin{array}{c}9,75 \times 10^{2} \\
\pm 6,82 \times 10^{2}\end{array}$ & $\begin{array}{c}5,83 \times 10^{2} \\
\pm 4,23 \times 10^{2}\end{array}$ \\
\hline
\end{tabular}


de todo o processo, desde a coleta da amostra até a confecção da lâmina de RID, uma vez que a única diferença entre estas amostras foi a etapa de desinfecção por UV e este processo, no tempo aplicado (cinco segundos), não destrói as formas infectantes dos protozoários.

O método de FM utilizado se mostrou uma eficiente opção, porém, não é recomendada para análise do esgoto bruto em função da grande quantidade de material particulado que rapidamente leva à saturação da membrana. Assim, a turbidez da amostra é um importante fator interferente no uso deste método, sendo às vezes necessária a troca de membranas para a filtração do volume total estipulado. Durante este estudo, a turbidez média das amostras do efluente foi de 5,47 NTU (mínimo de 3,13 e máximo de 13,3), sendo usada, em média, duas membranas para filtrar $1.200 \mathrm{~mL}$. Em apenas duas ocasiões foi possível filtrar $2.000 \mathrm{~mL}$ de efluente em uma única membrana (amostras com turbidez média de 2,8 NTU), porém, em uma amostra com turbidez de 13,3 NTU, foram utilizadas seis membranas para a filtração de 1.200 mL. O uso de muitas membranas implica em maior manipulação da amostra, pois são necessárias mais centrifugações para obtenção de um sedimento final único. Isso pode acarretar em maior chance de perda dos organismos, além de tornar o processamento mais trabalhoso e com um maior custo.

Considerando que a maioria das estações de tratamento de água (ETA), ou mesmo as ETE, já possuem o equipamento para a execução da técnica de FM para a análise de bactérias em amostras de água, o custo inicial de implantação do monitoramento destes protozoários é menor.

Embora o protocolo de FM seja conhecido pelos técnicos das empresas de tratamento de água e esgoto, o seu uso para detecção dos protozoários necessita de adaptações e treinamento para a identificação correta das formas de resistência encontradas nas amostras ambientais, uma vez que existe uma gama de estruturas semelhantes aos oocistos e cistos, induzindo identificações errôneas e acarretando resultados falso-positivos ou mesmo falsonegativos.

A etapa de purificação mediante separação imunomagnética (IMS) é amplamente utilizada para detecção de protozoários na análise de água. Seu uso na purificação de amostras de esgoto pode ser comprometido em função do grande número de material particulado e da quantidade de substâncias quelantes, tais como resíduos de fármacos, os quais podem interferir no desempenho desta etapa. A presença de compostos contendo carbono orgânico e mesmo de algumas formas de ferro, interfere na etapa de formação dos complexos microesferas anticorpos e epítopos dos oocistos e cistos durante o procedimento de IMS. A interação entre as espécies dissolvidas de ferro e a superfície de cistos e oocistos presentes na matriz, sem dúvidas, compromete a recuperação dos organismos (YAKUB; STADTERMAN-KNAUER, 2000; DIGIORGIO et al., 2002).

Em um estudo realizado com amostra de esgoto e o uso do IMS, os autores incluíram uma etapa de sedimentação espontânea, seguida de uma lavagem do sobrenadante previamente à etapa de purificação por IMS com o intuito de reduzir estes interferentes (IACOVISK et al., 2004). Porém, vale a pena ressaltar que, quanto mais etapas são adicionadas no processo, maior será a chance de perda de organismos durante a manipulação da amostra.

A etapa de visualização por RID é considerada imprescindível para análise destes protozoários em amostras ambientais, sendo 12 vezes mais eficiente que as técnicas comuns de coloração para oocistos e cistos (CLANCY et al., 1999). Este procedimento torna os resultados mais precisos, porém, devido à ocorrência de estruturas similares aos oocistos e cistos, como leveduras e algas, essa etapa deve ser realizada por um profissional apto, evitando, assim, resultados errôneos. A aquisição do kit com anticorpo monoclonal para a etapa de visualização pode ser considerada a parte mais onerosa da implantação dos métodos discutidos neste estudo. Vale ressaltar que outro tipo de amostra ambiental que deve ser analisado quanto à presença de patógenos é o lodo de esgoto condicionado. A disposição deste resíduo da ETE no solo como condicionador ou fertilizante agrícola tem-se mostrado como a opção mais econômica, porém, é necessário avaliar o risco desta aplicação, levando-se em consideração os problemas relacionados à saúde pública. Métodos para a análise deste tipo de amostra foram analisados no trabalho de Bonatti (2007).

De maneira geral, as técnicas de FM e de CC podem ser consideradas de baixo custo e fácil aplicabilidade, o que facilita a implantação de um monitoramento dos protozoários sendo, portanto, uma boa opção para as indústrias de tratamento de esgoto e as produtoras de água.

\section{Considerações finais}

Resultados laboratoriais são elementos importantes para a tomada de decisões na área de saneamento e, consequentemente, na saúde pública. Erros de resultados provenientes de análises microbiológicas podem induzir a sérios erros de planejamento e direcionamento de programas de controle das diversas enfermidades de veiculação hídrica.

A maioria dos ensaios com amostras de água já possuem metodologias padronizadas e validadas, além de diretrizes que devem ser seguidas pelos laboratórios de análises para assegurar a qualidade da detecção de parasitos de veiculação hídrica. Entre estas orientações está a periodicidade de experimentos que indiquem a sensibilidade dos métodos utilizados, a constante manutenção dos equipamentos utilizados, bem como atualização do pessoal 
envolvido nas análises. Esses cuidados devem ser tomados também para a análise de amostras de esgoto, começando por estudos de padronização e validação de metodologias. Esses cuidados visam assegurar a qualidade dos resultados e, assim, minimizar e promover resultados confiáveis, uma vez que o reuso de efluente de esgoto tem sido cada vez mais visto como uma possibilidade para o problema da escassez hídrica. As técnicas aplicadas neste estudo mostraram-se eficientes na detecção de cistos de Giardia spp. e oocistos de Cryptosporidium spp. presentes nas amostras de esgoto avaliadas, além de apresentarem baixo custo por análise.

\section{Referências}

AIYUK, S. et al. Anaerobic and complementary treatment of domestic sewage in regions with hot climates - A review. Bioresource Technology, v. 97, n. 17, p. 2225-2241, 2006.

BONATTI, T.R. Ocorrência de cistos de Giardia spp., oocistos de Cryptosporidium spp. e ovos da família Ascarididae em amostras de lodo de esgoto. 2007. Dissertação (Mestrado) - Universidade Estadual de Campinas, Campinas, 2007.

BOULTER-BITZER, J.I.; LEE, H.; TREVORS, J.T. Molecular targets for detection and immunotherapy in Cryptosporidium parvum. Biotechnology Advances, v. 25, n. 1, p.13-44, 2007.

BUKHARI, Z. et al. Immunomagnetic separation of Cryptosporidium parvum from source water samples of various turbidities. Applied and Environmental Microbiology, v. 64, n. 11, p. 4495-4499, 1998.

CANTUSIO NETO, R..; SANTOS, L.U.; FRANCO, R.M. Evaluation of activated sludge treatment and the efficiency of the disinfection of Giardia species cysts and Cryptosporidium oocysts by UV at a sludge treatment plant in Campinas, south-east Brazil. Water Science and Technology, v. 54 , n. 3, p. 89-94, 2006

CANTUSIO NETO, R. et al. Cryptosporidium spp. and Giardia spp. in samples of natural water from the Atibaia River, city of Campinas, São Paulo, Brazil. Water Science Technology, v.62, n. 1, p. 217-222, 2010

CLANCY, J.L. et al. USEPA Method 1622. Journal AWWA, v. 91, n. 9, p. 60-68, 1999.

DIAS Jr., O. Ocorrência de cistos de Giardia sp. e oocistos de Cryptosporidium sp. em águas superficiais e esgoto no município de Araras/SP. Dissertação (Mestrado) - Universidade Presbiteriana Mackenzie, São Paulo, 1999

DIGIORGIO, C.L.; GONZALEZ, D.A; HUITT, C.C. Cryptosporidium and Giardia recoveries in natural waters by using environmental protection agency method 1623. Applied and Environmental Microbiology, v. 12, p. 5952-5955, 2002.

FARIAS, E.W.C.; GAMBA, R.C.; PELLIZARI, V.H. Detection of Cryptosporidium spp. oocysts in raw sewage and creek water in the city of São Paulo, Brazil. Brazilian Journal Microbiology, v. 33, n. 1, p. 41-43, 2002.

FRANCO, R.M.B.; ROCHA-EBERHARDT, R.; CANTUSIO NETO, R. Occurrence of Cryptosporidium oocysts and Giardia cysts in raw water from the Atibaia river, Campinas, Brazil. Revista do Instituto de Medicina Tropical, v. 43, n. 2, p. 109-111, 2001.

$\mathrm{HACHICH}$, E.M. et al. Giardia and Cryptosporidium in source waters of Sao Paulo State, Brazil. Water Science Technology, v. 50, n. 1, p. 239 245, 2004

IACOVSKI, R.J.; BARARDI, C.R.M.; SIMÕES, C.M.O. Detection and enumeration of Cryptosporidium sp. Oocysts in sewage sludge samples from the city Florianópolis (Brazil) by using immunomagnetic separation combined with indirect immunofluorescence assay. Waste Management Research, v. 22, p. 171-176, 2004

LEAL, D.A.G. et al. First Report of Cryptosporidium spp. oocysts in Oysters (Crassostrea rhizophorae) and Cockles (Tivela mactroides) in Brazil. Journal Water Health,v. 6, n. 4, p. 527-532, 2008.

LI, D. et al. Infectivity of Giardia lamblia cysts obtained from wastewater treated with ultraviolet light. Water Research, v. 43, n. 12, p. 3037-3046, 2009

LINDQUIST, H.D.A. et al. Criteria for evaluation of proposed protozoan detection methods. Journal Microbiology Methods, v. 37, p. 33-43, 1999

MACPHERSON, C.N.L. Human behavior and epidemiology of parasitic zoonoses. International Journal Parasitology, v. 35, p. 1319-1331, 2005.

MONTEMAYOR, M. et al. Occurrence of Cryptosporidium spp. Oocysts in raw and treated sewage and river water in north-eastern Spain. Journal Applied Microbiology, v. 99, p. 1455-1462, 2005.

NITHIUTHAI, S. et al. Waterborne zoonotic helminthiases. Veterinary Parasitology, v. 126, p.167-193, 2004.

NORDGREN, J. et al. Prevalence of norovirus and factors influencing virus concentrations during one year in a full-scale wastewater treatment plant. Water Research, v. 43, n. 4, p. 1117-1125, 2009.

ORGANIZAÇÃO DAS NAÇÕES UNIDAS (ONU). Relatório do desenvolvimento Humano Cap 1, 27-74, 2006

OTTOSON, J. et al. Removal of viruses, parasitic protozoa and microbial indicators in conventional and membrane processes in a wastewater pilot plant. Water Research, v. 40, p.1449-1457, 2006 
ROBERTSON, L.J. etal. Giardia cysts and Cryptosporidium oocysts at sewage treatment works in Scotland, UK. Water Research, v. 34, p. 2310-2322, 2000

ROCKWELL, R.L. Giardia lamblia e giardiasis with particular attention to the Sierra Nevada, 2003. Disponível em < http://www.yosemit.org/ naturenotes/archive.htm>. Acesso em Janeiro de 2007.

ROSE, J.B.; HUFFMAN, D.E.; GENNACCARO, A. Risk and control of waterborne cryptosporidiosis. FEMS Microbiology Review, v. 26, p. 113123, 2002.
SANTOS, L.U. et al. Detection of Giardia spp. cysts and Cryptosporidium oocysts in activated sludge by ether clarification technique and sucrose flotation. Revista do Instituto de Medicina Tropical São Paulo, v. 46, p. 309-313, 2004.

YAKUB, G.P.; STADTERMAN-KNAUER, K.L. Evaluation of immunomagnetic separation for recovery of Cryptosporidium parvum and Giardia duodenalis from high-iron matrices. Applied and Environmental Microbiology, v. 66, p. 3628-363, 2000. 